\title{
Integrins in invasive growth
}

\author{
Cord Brakebusch, ${ }^{1,2}$ Daniel Bouvard, ${ }^{1}$ Fabio Stanchi, ${ }^{1}$ Takao Sakai, ${ }^{1}$ and Reinhard Fässler ${ }^{1,2}$ \\ ${ }^{1}$ Lund University Hospital, Department of Experimental Pathology, Lund, Sweden \\ ${ }^{2}$ Max Planck Institute for Biochemistry, Martinsried, Germany \\ Address correspondence to: Cord Brakebusch, Max Planck Institute for Biochemistry, \\ Department of Molecular Medicine, Am Klopferspitz 18a, 82152 Martinsried, Germany. \\ Phone: 49-89-8578-2466; Fax: 49-89-8578-3777; E-mail: brakebus@biochem.mpg.de. \\ J. Clin. Invest. 109:999-1006 (2002). DOI:10.1172/JCI200215468.
}

Interactions between tumor cells and the ECM strongly influence tumor development, affecting cell proliferation and survival, as well as the ability to migrate beyond the original location into other tissues to form metastases. Many of these interactions are mediated by integrins, a ubiquitously expressed family of adhesion receptors. Integrins are essential for cell attachment and control cell migration, cell cycle progression, and programmed cell death, responses that they regulate in synergy with other signal transduction pathways.

This large group of transmembrane proteins is formed from $18 \alpha$ and $8 \beta$ subunits, which dimerize to yield at least 24 different integrin heterodimers, each with distinct ligand binding and signaling properties. With their extracellular domain, integrins can bind to different ECM molecules, such as collagens and laminins, or to cellular receptors, such as VCAM-1. Their intracellular domains connect directly or indirectly to the actin cytoskeleton, thus linking the cytoskeleton to the ECM. Integrins also serve as bidirectional signaling receptors, inducing changes in protein activities or gene expression in response to ligand binding, while also modulating adhesive affinity on the cell surface in response to changes in cellular physiology. Here, we describe how integrins affect migration, proliferation, and survival of both transformed and normal cells, and we discuss how they modulate invasive growth in vivo. Throughout, we stress that many of these functions are restricted to particular cell types and may be altered upon transformation.

\section{Integrin avidity and affinity changes in cell migration}

Cell migration is essential not only for tissue infiltration and the formation of metastases, but also for nonpathological processes, such as angiogenesis and leukocyte extravasation. In order to migrate, a cell has to pass through a sequence of distinct processes. Migration is initiated by cell polarization and the formation of membrane protrusions at the leading edge. Integrins fix cellular protrusions to the ECM, interact with the actin cytoskeleton, and trigger the association of many different signaling molecules at the so-called focal contacts. Thereafter, integrin signals stimulate cell contraction, which allows the movement of the cell body on the adhesive contacts. Finally, the rear of the cell detaches from the substratum by inactivation of the integrins and disassembly of the adhesion complexes. Integrins further facilitate cell movement through the tissue by activating ECM-degrading enzymes. Although these basic mechanisms are considered to be similar among the various types of migratory cells, there are also clear distinctions, since, for example, fibroblasts are about 3-20 times more adhesive than nonactivated leukocytes, move 10-60 times more slowly, and have a different cytoskeletal organization. These differences in the migration mechanism might explain at least some of the known cell-specific effects of integrins on cell migration.

Integrin-mediated cell attachment depends not just on the expression of these receptors in a given cell type, but also on their affinity for various ligands and on their lateral mobility within the plasma membrane, which allows the formation of high-avidity clusters. Integrins adopt low- and high-affinity conformations, which can be distinguished by their binding to soluble ligands or by conformation-specific antibodies. High-affinity binding, which is important for the firm attachment of the leading edge to the ECM, can result from the binding of regulatory intracellular molecules to the cytoplasmic domains of the $\alpha$ and $\beta$ subunits. Overexpression in osteosarcoma cells or normal human fibroblasts of chimeric proteins consisting of the $\beta 1, \beta 3$, or $\beta 5$ intracellular domains, fused to the extracellular and transmembrane part of the IL-2 receptor, significantly reduces the affinity of the endogenous $\beta 1$ integrins (1). This effect is most likely caused by sequestration of intracellular molecules that would otherwise bind the cytoplasmic domain of the integrin $\beta$ subunits and alter the integrins' extracellular conformation. Such an effect, called transdominant inhibition, has also been reported for $\alpha 2$ integrin cytoplasmic domains (2). More than 20 proteins are currently known to bind intracellular domains of integrin subunits (3), but it is not known which of 
them are involved in the modulation of integrin affinity. Potential candidates for such regulation are ICAP-1 and TAP-20, which decrease, and $\beta 3$-endonexin, which increases integrin-mediated adhesion in cells overexpressing these molecules. Intracellular signaling mechanisms, for example phosphorylation of ICAP-1 by CaMKII, are suggested to modulate the binding of these molecules to integrin and thereby the affinity state.

Cell attachment can be induced not only by affinity regulation, but also by clustering of integrins, which leads to increased adhesive avidity. Such clusters are present in focal adhesions, readily detectable cellmatrix contacts that have been extensively studied in cultured fibroblasts. Increased lateral mobility of integrins in the membrane might also be important for efficient ligand binding. In leukocytes, even prior to ligand binding, integrins seem to be associated with the actin network, which constrains integrin mobility and ligand binding. Releasing this contact by proteolytic digestion of the integrin connections with the cytoskeleton results in a rapid increase in lateral mobility of integrins, which increases the chance of ligand encounters and facilitates integrin aggregation into high-avidity clusters (4).

\section{Signaling pathways regulating integrin affinity}

Growth factor and chemokine signaling can also modulate the affinity and avidity of integrins. In particular, upregulation of integrin activity by growth factors often depends on phosphatidylinositol 3-kinase (PI3-K) activation. Thus, in mast cells, activation of PI3-K by FceRI, c-kit, or PDGF-R increases the affinity of $\alpha 5 \beta 1$ integrin. In metastatic breast cancer cells, increased cell adhesion and migration upon stimulation of EGF-R or erbB3 are also dependent on PI3-K. Integrin avidity is upregulated in carcinoma cells by treatment with HGF (see Danilkovitch-Miagkova and Zbar, this Perspective series, ref. 5), but not with EGF. This increased integrin avidity is dependent on PI3-K and promotes invasive growth of these cells, suggesting an important role of integrin avidity regulation in metastasis (6).

Signaling initiated by chemokine receptors can induce a rapid and transient upregulation of integrin affinity, which is important for the tight adhesion of leukocytes to the endothelium during their extravasation into inflamed tissues. At least in lymphocytes, this activation is independent of PI3-K and involves activation of RhoA (4). Remarkably, chemokines can independently regulate the affinity and avidity of different integrins within the same cell. In eosinophils, the chemokines RANTES and monocyte chemoattractant protein-3 induce a transient upregulation of $\alpha 4 \beta 1$ avidity and a long-lasting affinity increase of $\alpha \mathrm{M} \beta 2$ (Mac-1). Chemokines such as secondary lymphoid tissue cytokine, EBI1-ligand chemokine, and stroma cellderived factor $1 \alpha$ stimulate integrin motility in lymphocytes through the coordinated action of cytosolic proteases and PI3-K. In contrast, PMA-stimulated integrin mobility is dependent on proteases, but independent of PI3-K (4).
The Ras and Rho GTPases can also regulate integrin affinity, although their effects are cell type- and integrin-specific. In Chinese hamster ovary $(\mathrm{CHO})$ cells, for example, $\mathrm{H}$-Ras inhibits activation of $\beta 1$ and $\beta 3$ integrins, while in a pro-B cell line it increases $\alpha$ L $\beta 2$ (LFA-1) activity in a PI3-K-dependent manner (7). R-Ras promotes integrin activation in myeloid cells but has no effect in a lymphoid cell line (7). The integrin-specificity of Ras effects is also nicely demonstrated in T47D cells, where R-Ras promotes migration mediated by $\alpha 2 \beta 1$ but not by $\alpha 5 \beta 1$ (2). Ras signaling can affect many signaling cascades, including the activation of Erk and PI3-K. The integrin activating functions of both H-Ras and R-Ras require PI3-K activation.

Recently, Rap1 was shown to activate integrins on lymphoid and endothelial cells by inducing a highaffinity conformation $(7,8)$. Overexpression of constitutively active Rac1 in lymphoid cells increases integrin affinity (7). In mammary epithelial cells, however, neither Rac1 nor Cdc42 induces obvious changes in integrin avidity or affinity, although they promote integrinmediated motility and invasiveness through PI3-K.

Several reports have demonstrated cross-talk between integrins, in which signaling by one integrin influences the affinity or avidity of others. For example, stimulation of $\alpha$ IIb $\beta 3$ signaling has been shown to downregulate $\alpha 2 \beta 1$ - and $\alpha 5 \beta 1$-mediated adhesion.

A few reports have also shown that direct interaction of secreted molecules with the extracellular domain of integrins can influence integrin affinity. Galectin-8, for example, a secreted galactoside-binding protein, binds $\beta 1$ integrins and induces a conformational change that decreases their affinity for other ligands (9).

\section{Detachment of the cell rear}

Downregulation of integrin binding at the rear is required for directed movement. This process is poorly understood, but it is clear that cell migration can be inhibited by locking integrins into a high-affinity state by mutations or activating antibodies. Detachment of the cell rear also requires a contraction of the cell body. Activation of RhoA and its downstream targets ROCKI and II, which are essential for the detachment of migrating leukocytes at the cell rear, mediates both decreased adhesion by $\alpha 4 \beta 1$ and $\beta 2$ integrins and increased intracellular force generation, resulting from stimulation of myosin light chain phosphorylation (10). Migrating fibroblasts can leave integrins, but not integrin-associated proteins like vinculin and $\alpha$-actinin, in fibrous structures behind the cell, suggesting a disruption of the integrin-cytoskeleton connection (11). This might be caused by contraction or by intracellular proteolytic cleavage of integrins and other focal adhesion proteins and a concomitant disintegration of focal adhesions. Activation of Src may play a pivotal role in the regulation of focal adhesion turnover during migration, since the constitutively active v-Src oncoprotein is located in focal adhesions, where it induces focal adhesion kinase (FAK) phosphorylation, calpain-mediated degradation of focal adhesion proteins, and, eventually, disassembly of focal adhesions during transformation (12). It is conceivable that growth factors 
like EGF promote cell migration by reducing cell adhesion and the number of focal adhesions through the same mechanism. Cleavage products of FAK or the focal adhesion-associated docking protein HEF-1 can induce cell rounding, which is important for cell division and might also be involved in migration (13).

All of the detachment mechanisms discussed above appear to be sensitive to $\mathrm{Ca}^{2+}$ transients. Migrating fibroblasts have increased $\mathrm{Ca}^{2+}$ levels in the rear, perhaps as a consequence of stretch-activated calcium channels opening in response to cell contraction at the trailing edge. In neutrophils, $\mathrm{Ca}^{2+}$-induced activation of calcineurin disrupts interactions between $\alpha v \beta 3$ and the cytoskeleton, allowing detachment of the rear. $\mathrm{Ca}^{2+}$ transients are also essential to release $\alpha 5 \beta 1$-mediated attachment of neutrophils to fibronectin (14). This latter mechanism, however, is independent of calcineurin and may involve modulation of integrin affinity or of cellular contractility. $\mathrm{Ca}^{2+}$ influx may also lead to the activation of $\mu$-calpain, which is proposed to sever the connection between the integrin and the cytoskeleton proteolytically.

During rear detachment, unbound integrins accumulate at the trailing edge. Endocytosis of these detached integrins and their transport to the leading edge are thought to be needed to prevent integrin depletion at the front (14). Such endocytotic transport of green-fluorescent protein-tagged $\alpha 5$ integrin subunits has indeed been observed in migrating fibroblasts (11). Integrin molecules observed in endocytotic vesicles are not attached to $\alpha$-actinin or vinculin, as they are in the focal contacts prior to endocytosis. In monocyte chemoattractant protein-7 breast cancer cells, protein kinase $\mathrm{C} \alpha$ participates in the recycling of the activated pool of $\beta 1$ integrins (15).

\section{Integrin signaling in migration}

Integrin signaling promotes cell migration by inducing changes in the cytoskeletal organization and by increasing cellular contractility (Figure 1). Activation of FAK plays a prominent role among the different integrin signaling pathways which affect migration. FAK is a non-receptor tyrosine kinase that is indirectly and perhaps also directly associated with integrins in focal contacts. Ligand binding to integrins leads to phosphorylation of FAK on at least seven different tyrosines in vivo, allowing the interaction of FAK with Src, Grb2, and $\mathrm{PI} 3-\mathrm{K}$, and also promoting the phosphorylation of associated proteins, such as Cas and paxillin. FAK therefore functions as an important adaptor molecule that recruits various other signaling molecules to focal contacts. FAK is also a target for tyrosine phosphorylation induced by growth factor receptors. For this reason, FAK serves as an important integration point of growth factor and integrin signaling with respect to cell migration (16). Experiments in which FAK mutants are expressed in otherwise FAK-deficient fibroblasts have shown that FAK kinase activity and FAK autophosphorylation of tyrosine 397 are required for integrinstimulated cell migration, whereas FAK's association with paxillin is dispensable.
FAK can promote cell movement by activating PI3-K and regulators of cytoskeletal dynamics like Rac1. Autophosphorylation of FAK on tyrosine 397 allows this molecule to bind and activate PI3-K, which can then influence integrin affinity and avidity, as discussed above. In addition, PI3-K contributes to the activation of Rac1, which mediates membrane ruffling. In adherent but not in suspended cells, activated Rac interacts with and activates p21-activated kinase (PAK), which can then stimulate migration by increasing the turnover of focal adhesions (17). Tyrosine-phosphorylated FAK also promotes Rac activation via an alternative pathway involving the adaptor proteins $\mathrm{p} 130^{\text {Cas }}$ and $\mathrm{Crk}(18,19)$.

Integrin signaling also stimulates $\mathrm{Cdc} 42$ and RhoA activity and facilitates the interaction of these Rhofamily GTPases with their downstream targets. These GTPases cooperate and influence each other's activity in a complex, cell-specific manner. In astrocytes, integrin-dependent stimulation of Cdc42 via Src-like kinases is crucial for cell polarity, protrusion formation, and migration (20). In $\mathrm{CHO}$ cells, $\alpha 5 \beta 1$-mediated activation of Rac1 and Cdc42 is maximal already at intermediate fibronectin levels, whereas Rho activity continues to increase with increasing fibronectin levels (21). Since Rac1 and Cdc42 promote, but RhoA inhibits, membrane protrusion, increased integrin-mediated Rho GTPase activation halts migration in these cells preferentially at high fibronectin concentrations.

Recently, Chen et al. (22) found that the cytoplasmic tyrosine kinase Etk, which is highly expressed in migratory cells including endothelial cells and metastatic cell lines, is activated by integrin signaling. Integrin-mediated activation and autophosphorylation of FAK lead to interaction of FAK with the pleckstrin domain of Etk, and subsequent phosphorylation and activation of Etk. Etk may play an important role in migration, since changes in its expression directly correlate to changes in migration. Downstream targets of Etk mediating such a promigratory role are not yet known.

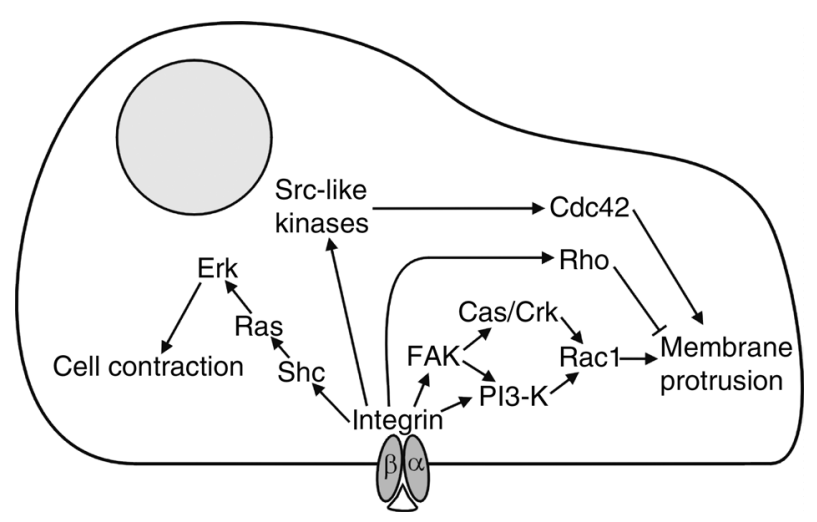

Figure 1

Major integrin-linked signaling pathways affecting migration. Integrin signaling stimulates Rho GTPases and thereby induces changes in the cytoskeletal organization that are required for cell migration. Ligand binding to integrins also promotes cell contractility through the activation of the tyrosine kinase Erk. 
Integrin-triggered activation of Erk can contribute to migration via phosphorylation of MLCK, leading to increased phosphorylation of MLC and cell contraction (23). Erk activation furthermore leads to changes in gene expression, which have been suggested to promote migration. After integrin engagement or stimulation of $\mathrm{v}$-Src, active Erk can also be targeted to newly forming focal adhesions, where it may phosphorylate focal adhesion proteins and influence cytoskeletal organization and migration (24). Erk can be activated via the interaction of FAK with Grb2. This pathway, however, apparently does not play a major role in integrin-mediated migration, since mutation of the Grb2-binding tyrosine 925 of FAK does not affect cell migration (18). Certain integrins can induce Erk activation independently of FAK via the Shc/Grb2/Sos/Ras cascade. $\alpha 1 \beta 1, \alpha 5 \beta 1$, and $\alpha v \beta 3$ integrins activate this alternative pathway via an interaction of their $\alpha$ subunit with the membraneassociated protein caveolin, which helps define a distinctive membrane subdomain in which Shc becomes phosphorylated by the Src-like kinase Fyn (25).

\section{Integrins and matrix metalloproteinases}

Cell migration in vivo is often facilitated by a partial destruction of the surrounding ECM. Such degradation is catalyzed by matrix metalloproteinases (MMPs), a family of more than 20 substrate-specific zincdependent endoproteases, most of them soluble, but some of them transmembrane proteins. MMP activity, which is controlled by regulated expression, by proteolytic activation of inactive precursors (zymogens), and by the expression of a family of inhibitors, is crucial for tumor invasion, metastasis, and angiogenesis. Integrins can regulate the expression and activation of MMPs and can guide them to their targets by simultaneous binding of MMPs and ECM molecules.

Various integrin-induced signaling pathways are involved in the control of MMP expression. In collagen gels, for example, $\alpha 1 \beta 1$ - and $\alpha 2 \beta 1$-dependent expression of MMP-13 requires p38 activity and is inhibited by Erk. In other settings, overexpression of the integrinlinked kinase (ILK) can result in AP-1-dependent expression of MMP-9 and an invasive phenotype. Finally, stimulation of the $\alpha 3 \beta 1$-tetraspanin complex on mammary epithelial cells upregulates the expression of MMP-2 and increases their invasive potential. Some of these transcriptional effects might be mediated by the transcription factor Cas-interacting zinc finger protein (CIZ), which can shuttle between the focal adhesions and the nucleus (26). CIZ activates the expression of several MMPs, and its effect is enhanced in the presence of Cas, which mediates some of the effects of FAK as mentioned above. However, integrins can also reduce the expression of certain MMPs. Deletion of the mouse gene for $\alpha 1$ integrin, for example, leads to a marked increase of MMP-7 and MMP-9 in the serum (27). MMP activation by integrins can also occur indirectly, through the increased expression of zymogen-activating proteases. Signaling by $\beta 1$ integrin on ovarian carcinoma cells stimulates MMP-2 activity, perhaps by upregulating the activating protease MT1-MMP.
Yet another mechanism for modulating MMP function involves the simultaneous binding of integrins to ECM molecules and to MMPs, thus bringing the proteases close to their target. Complex formation of integrins and MMPs is crucial for wound healing and angiogenesis, as has been shown in basal keratinocytes of injured skin, where $\alpha 2 \beta 1$ integrin not only induces MMP-1 but also forms a complex with pro-MMP-1 (28). Similarly, in endothelial cells, $\alpha v \beta 3$ induces the production of MMP-2 and subsequently interacts with the newly synthesized MMP-2 to promote vascular invasion. Without binding to $\alpha v \beta 3$, MMP- 2 is not functional (29). Inhibition of the $\alpha v \beta 3-M M P-2$ interaction by small organic compounds or viral vector-encoded peptides might therefore allow for reduced metastasis without blocking the binding of other molecules to $\alpha v \beta 3$.

\section{Integrin-mediated proliferation}

The cell cycle is controlled by cyclin-dependent kinases (cdks). These proteins are constitutively expressed, but their activity is regulated by binding to cyclins and by the action of cdk inhibitory proteins (CKIs). Progression through the $\mathrm{G} 1$ phase is controlled by cdk 4 and cdk6, which interact with cyclin D1, and by cdk2, which binds to cyclin E. The CKIs p21 cip1 and p27kip1 inactivate cdk2. Cdks are responsible for the phosphorylation of $\mathrm{Rb}$, which induces other cell cycle proteins including cyclin A. Association of cyclin A with cdk2 initiates then the G1/S transition.

Integrin signaling can regulate the G1/S transition in tight synergism with growth factors (Figure 2). Here again, however, different cell types require distinct integrins and growth factors for proliferation. In endothelial cells, for example, signaling of both bFGF and $\alpha v \beta 3$ is required for cell cycle progression, whereas in fibroblasts bFGF can also synergize with $\beta 1$ integrins (30). Integrin-mediated proliferation depends on the activation of the Erk pathway, which controls expression of cyclin D1 and p21 cip. Integrins can induce Erk via FAK or via caveolin, Fyn, and Shc signaling, both converging on the Ras/Erk cascade.

Since growth factor receptors also activate Erk via the Ras pathway, it was initially unclear why both integrin and growth factor receptor signaling are necessary for proliferation. Recent data, however, indicate that only combined signaling of both pathways results in the appropriate kinetic and extent of Erk activation. Growth factors activate Erk strongly but transiently, which is not sufficient for the induction of cyclin D1. On the other hand, integrin-mediated activation of Erk is too weak to stimulate expression of cyclin D1. Only cells attached to the ECM via integrins show a sustained and robust activation of Erk in response to growth factors, which is necessary to induce cyclin D1. In fibroblasts, strong sustained (5-20 hours), but not transient $(<1$ hour) or weak, Erk activation results in the expression of a distinct repertoire of fos and jun proteins, which might be responsible for the differential effects on cyclin D1 expression (31). Indeed, the Cyclin D promoter contains an AP- 1 site, which is 


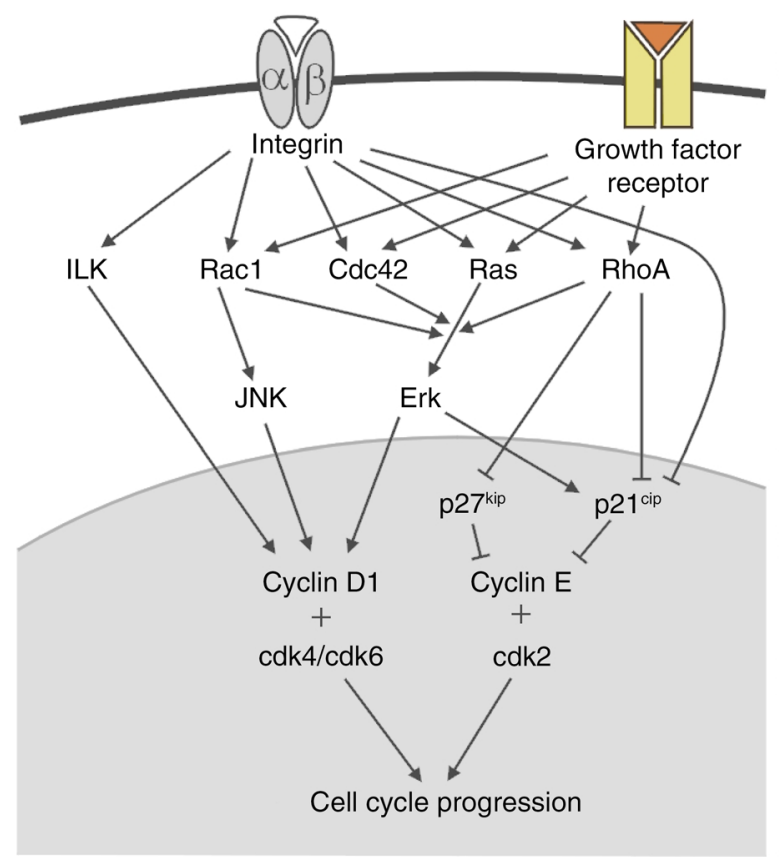

Figure 2

Integrin control of proliferation. Synergistic activation of Ras and Rho GTPases by integrins and growth factors is necessary for cell cycle progression. Activation of Rac1, Cdc42, and RhoA can facilitate activation of Erk via Ras. Erk, as well as JNK and ILK, can induce expression of cyclin D1, thus promoting cell cycle progression. In addition, integrins can promote proliferation by inhibiting cell cycle inhibitors like p21 cip and p27kip.

recognized by several jun/fos heterodimers, and a CRE site, to which heterodimers of jun/fos and certain ATF/CREB family members might bind.

A second integrin-dependent proliferation checkpoint exists at the level of the cdk inhibitors. Thus, transient, high-level Erk activation increases p21 $1^{\text {cip }}$ expression, which inhibits cyclin $\mathrm{E} / \mathrm{cdk} 2$. Integrindependent signals, but not Erk, downregulate $\mathrm{p} 21^{\text {cip }}$ in mid- to late G1, allowing cell cycle progression.

The molecular mechanisms by which integrin and growth factor receptor signaling pathways synergize to influence Erk activation are not yet clear, and several possible models should be considered. First, integrin signaling can influence the phosphorylation state of EGF and PDGF receptors as well as their association with signaling molecules such as SHP-2, Ras-GAP, IRS-1, and PI3-K. Interestingly, FAK activation is not required for the integrin-mediated increase of EGF-R phosphorylation. Alternatively, integrins may facilitate signaling of growth factor receptors via the Ras cascade. In some cells, for example, growth factor activation of Raf or MEK, two serine/threonine kinases upstream of Erk, is dependent on cell adhesion. This effect might be due to the integrin-mediated activation of PAK, which phosphorylates both Raf and MEK (32). Such cross-talk between integrin and growth factor signaling can be essential for the transformation of cells. The malignant behavior of a breast cancer cell line in a three-dimensional culture is crucially dependent on both integrin and EGF-R signaling, which together result in sustained Erk activation. Inhibition of integrins, EGF-R, or Erk leads to growth arrest and normal breast tissue morphogenesis. Interestingly, these effects are not seen in two-dimensional cell cultures, suggesting that the connections between signaling pathways depend on the spatial organization of the cytoskeleton.

Integrin-mediated cell cycle control has also been demonstrated in vivo. Thus, Faraldo et al. (33) have found that inhibition of integrin signaling in mice using a dominant negative integrin fusion protein reduces proliferation of mammary cells and attenuates Shc, Erk, and JNK activation without perturbing FAK activity.

\section{Rho GTPases in integrin-mediated proliferation}

In fibroblasts, but not in endothelial cells, the synergism between integrin and growth factor signaling is dependent on cell spreading, indicating an important role of the cytoskeleton. Rho GTPases might be key players in this regulation since they are activated by growth factors and, weakly, by integrins, and since they are pivotal for the organization of the cytoskeleton. Rho GTPases are crucial for cell cycle progression, as seen in the impairment of growth factor-induced production of cyclin D1 following treatment of adherent cells with the Rho inhibitor toxin A. RhoA downregulates the transcription of p21 cip and promotes degradation of the CDK inhibitor p27kip1 by stimulation of cyclin $\mathrm{E} / \mathrm{cdk} 2$ activity. Binding to fibronectin can induce cyclin D1 and suppress p21 cip in a Rho-dependent manner (34). Activation of RhoA and Cdc42 contributes to the integrin-mediated activation of Erk2. Activated Rac1 can induce cyclin D1 expression in serum-starved cells correlating with the activation of PAK-1, JNK, and NF- $\kappa B$. Furthermore, in endothelial cells Rac1 can facilitate translation of cyclin D1 mRNA upon activation by $\alpha 5 \beta 1$ integrin (35). Here, the integrininduced Rac activation depends on Shc, FAK, and PI3-K signals, which converge to regulate the guanine nucleotide exchange factor Sos.

Other integrin-dependent proliferation pathways have also been documented. Integrin and growth factor receptors both activate $\mathrm{PI} 3-\mathrm{K}$, which downregulates p27kip and contributes to the induction of cyclin D1. These effects are at least partly mediated by the PDK-1-dependent stimulation of the p70 S6 kinase, a key regulator of cell growth that controls protein translation and induces cyclin D3 expression (36). Many human tumors carry mutations in proteins of the PI3-K signaling pathway, underlining the importance of this mechanism in the prevention of malignant proliferation. In some cells, integrin-dependent activation of JNK also contributes to proliferation (37). JNK phosphorylates several members of the jun/fos family, causing increased transcriptional activity and reduced degradation of AP-1 proteins, which are important for the induction of cyclin D1. Finally, integrins might induce cell cycle progression by ILK, which is proposed to induce cyclin D1 expression by activating the transcription factor LEF-1. 


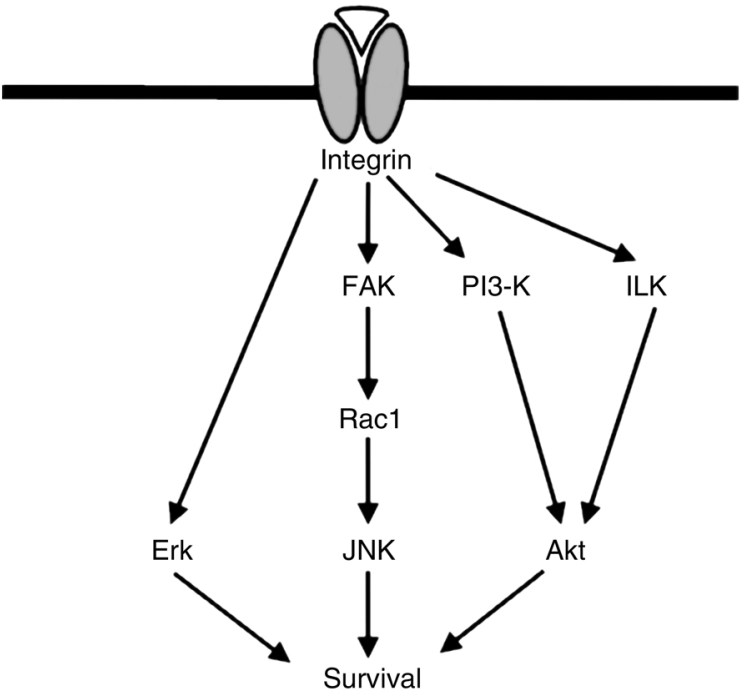

Figure 3

Integrin control of cell survival. Integrin activation of Akt via PI3-K or ILK plays an important role in the antiapoptotic effect of integrin-mediated adhesion. Activation of Erk and JNK likewise contributes to increased cell survival.

\section{Integrins and apoptosis}

Apoptosis or programmed cell death regulates the lifespan of normal cells and eliminates cells after contact with certain toxic insults or following their detachment from the ECM. This last form of apoptosis is also called "anoikis." Reduced apoptosis can contribute to tumorigenesis, since it helps tumor cells to escape natural elimination.

Apoptosis is initiated by either the signaling of death receptors or the release of cytochrome $c$ from the mitochondria, which then activates a cascade of intracellular proteases of the caspase family. The first pathway is controlled by the expression of death receptors and soluble or inactive "decoy" receptors. Induction of programmed cell death through the latter pathway is regulated especially by the balance of pro- and antiapoptotic members of the Bcl-2 family. Although anoikis is initiated by cell detachment, increased death receptor signaling also contributes to cell death. Integrin signaling can modulate apoptotic induction, leading in most cases to increased, but sometimes also to decreased, survival. Integrins can promote survival by activating PI3-K, ILK, Erk, and JNK (Figure 3). Integrin-mediated activation of PI3-K can trigger several antiapoptotic mechanisms. Activated PI3-K produces $\mathrm{PI}(3,4,5) \mathrm{P} 3$ and $\mathrm{PI}(3,4) \mathrm{P} 2$, which promote the relocation of Akt to the plasma membrane and stimulate its phosphorylation.

Akt blocks apoptosis by phosphorylating, and thus inactivating, a number of proapoptotic molecules, including the Bcl-2 family member Bad, caspase-9, and transcription factors of the forkhead family. Akt inhibits apoptosis via different pathways. It can also activate NF- $\kappa B$, via phosphorylation of $I \kappa B$, and thereby induce the expression of a set of survival factors, such as osteoprotegerin in endothelial cells.
Finally, it prevents the release of cytochrome $c$ from the mitochondria by an unknown mechanism. In $\mathrm{CHO}$ cells, integrin-mediated activation of PI3-K and $\mathrm{Akt}$, and upregulation of antiapoptotic $\mathrm{Bcl}-2$, are dependent on Shc and FAK-mediated Ras activation, but not on Erk (38).

Inhibition of $\beta 1$ integrin function in the mammary gland in vivo using a dominant negative receptor leads to increased cell death and reduced phosphorylation of Akt and the Akt substrates Bad and the forkhead transcription factor FKHR. FAK phosphorylation is normal under these conditions (33), indicating that integrinmediated activation of PI3-K in this tissue is independent of FAK, although it may be influenced by growth factor signaling, as has been described for mammary cells in culture. Some evidence suggests that the serine/threonine kinase ILK is activated after integrin binding and directly binds and phosphorylates Akt (39). In prostate carcinoma cells lacking the tumor suppressor PTEN, ILK is constitutively active and contributes to the survival of these cells.

Elicitation of Erk activity by integrins can prevent apoptosis, an effect that is limited to particular cell types and in some cases requires the additional activation of Rac1 by the FAK/Cas/Crk pathway (40). For example, in intestinal epithelial cells, $\alpha 2 \beta 1$-mediated Erk activation does not prevent apoptosis induced by serum deprivation (41). Integrin-mediated activation of JNK inhibits apoptosis of fibroblasts in the absence of growth factors (42). This effect is dependent on FAK, but independent of Akt and Erk. In the presence of growth factors, however, integrins prevent apoptosis of fibroblasts by FAKmediated activation of PI3-K and Akt.

Loss of these integrin-dependent survival signals after cell detachment can result in apoptosis. Detachment also leads to direct changes in the cytoskeleton and release of the proapoptotic molecule $\mathrm{Bmf}$, which is normally bound to the cytoskeleton (43), an event that is considered to help initiate apoptosis, since it occurs before caspase activation. Furthermore, unligated integrin can transmit apoptosis-stimulating signals. Overexpression of unligated, but not of ligand-bound, $\alpha 5 \beta 1$ integrin can induce apoptosis in a variety of detached human cancer cells.

As in migration and proliferation, the antiapoptotic functions of integrins depend on the context of other signaling pathways. Integrin signaling can enhance the survival effect of growth factors by facilitating downstream signaling events, as has been shown for $\alpha 6 \beta 1$ on oligodendrocytes and for $\alpha 5 \beta 1$ on intestinal epithelial cells and on mammary epithelium (41). On the other hand, signaling by the growth factor receptor c-erbB2 in a mammary epithelial cell line reduces the affinity of $\alpha 2 \beta 1$ integrin, decreases integrin-mediated survival signals, and results in apoptosis when the cells are growing on collagen (44). The tumor suppressor p53 modulates survival signals provided by $\alpha 6 \beta 4$ integrin in dramatic ways. $\alpha 6 \beta 4$ induces caspase-mediated inactivation of Akt and apoptosis in carcinoma cells expressing wild-type p53, while it promotes Akt-dependent survival in the absence of p53 (45). 
In endothelial cells, shear stress induces the upregulation of $\alpha 5 \beta 1$ integrin expression and inhibits programmed death of these cells. Interestingly, abrogation of shear stress causes apoptosis mediated by an autocrine loop: It induces the secretion of thrombospondin, which binds to $\alpha v \beta 3$, which acts as a death receptor. In neutrophils as well, integrins can have a proapoptotic role, since attachment via $\beta 2$ integrins renders the cells susceptible to TNF- $\alpha$-induced apoptosis.

\section{Integrins and invasive growth in vivo}

Many antibody and RGD peptide inhibition studies have suggested an important role for integrins in invasive growth during development. Analyses of mice lacking integrins, however, often fail to confirm such a role. For example, $\alpha v \beta 3$ integrin has been shown to be essential for angiogenesis, although deletion of the mouse gene for the $\alpha$ v subunit does not impair angiogenesis. These discordant results might be explained by side effects of the antibody/peptide treatments or, alternatively, by compensatory mechanisms that are activated in the mutant mice, such as the upregulation of other, functionally similar molecules. Defects in cell migration have been described during the development of animals lacking integrins. Murine primordial germ cells lacking $\beta 1$ integrin, for example, show impaired migration to the gonads. Interestingly, in Drosopbila, the PS1 integrin is required on tracheal cells of the visceral branch for normal migration of tracheal cells on the visceral endoderm; the interacting PS2 integrin is required on the visceral endoderm in the same migratory process (46).

A plethora of experimental evidence documents the importance of integrins in tumor progression, invasive growth, and metastasis. Using $\beta 1$-null tumor cells, for instance, it has been shown that $\beta 1$ integrin promotes but is not essential for metastasis. Mutations of the intracellular domain of the $\beta 1$ integrin chain differentially affect cell adhesion, invasion, and metastasis, indicating that integrin signaling is important for invasion. Different observations suggest an important role of $\alpha 6 \beta 4$ integrin in tumorigenesis. Expression of $\alpha 6 \beta 4$ integrin in $\beta 4$-deficient tumor cells increases their invasiveness via a PI3-K-dependent pathway. Furthermore, different tumor types maintain or even increase their levels of $\alpha 6 \beta 4$ expression.

It is often difficult to demonstrate a positive or negative correlation between tumor progression and integrin expression. This is due to the heterogeneity of tumors and to the fact that changes in the expression level of a single integrin subunit always have to be judged against the background of the expression levels of all other integrins, in addition to the activation status of growth factor and cytokine signal transduction pathways that modulate and synergize with integrin function. Although integrin-mediated binding events are essential for invasive growth, the migratory behavior of tumor cells is modulated by cytokines and growth factors to such a degree that the contribution of changes in integrin expression is often not obvious (47). As an example, $\alpha 2 \beta 1$ integrin expression seems to promote invasive growth in pancreatic carcinomas and rhabdomyosarcomas but is strongly downregulated in bladder, breast, and colon cancer.

There is strong evidence that integrins are involved in the resistance of tumor cells to chemotherapy-induced apoptosis. Adhesion of small-cell lung cancer cells to ECM molecules protects them from chemotherapeutic, apoptosis-inducing agents (48). This effect is mediated by $\beta 1$ integrins, which activate phosphotyrosine kinases in response to chemotherapy-induced DNA damage. In human myeloma cells, overexpression of $\alpha 4 \beta 1$ results in increased drug resistance. Finally, $\beta 1$ integrin-mediated activation of PI3-K in breast cancer cells increases the resistance of tumor cells to apoptosis-inducing drugs (49).

Integrins thus clearly offer attractive drug targets to fight tumor growth and metastasis and drug resistance, but the variability of their functions in different cell types is daunting. Much more will need to be learned about integrin function, cross-talk with other signaling pathways, and tumor-specific roles to ensure that such drugs will be effective and safe.

\section{Acknowledgments}

We thank Michael Dictor, Martin Pfaff, and Kristiina Vuori for critically reading the manuscript. We apologize that, due to space limitations, we could not cite primary references for all the work mentioned and had to omit many interesting contributions to the field. D. Bouvard is supported by a Marie Curie fellowship.

1. Mastrangelo, A.M., Homan, S.M., Humphries, M.J., and LaFlamme, S.E. 1999. Amino acid motifs required for isolated $\beta$ cytoplasmic domains to regulate 'in trans' $\beta 1$ integrin conformation and function in cell attachment. J. Cell Sci. 112:217-229.

2. Keely, P.J., Rusyn, E.V., Cox, A.D., and Parise, L.V. 1999. R-Ras signals through specific integrin $\alpha$ cytoplasmic domains to promote migration and invasion of breast epithelial cells. J. Cell Biol. 145:1077-1088.

3. Liu, S., Calderwood, D.A., and Ginsberg, M.H. 2000. Integrin cytoplasmic domain-binding proteins. J. Cell Sci. 113:3563-3571.

4. Constantin, G., et al. 2000. Chemokines trigger immediate $\beta 2$ integrin affinity and mobility changes: differential regulation and roles in lymphocyte arrest under flow. Immunity. 13:759-769.

5. Danilkovitch-Miagkova, A., and Zbar, B. 2002. Dysregulation of Met receptor tyrosine kinase activity in invasive tumors. J. Clin. Invest. 109:863-867. DOI:10.1172/JCI200215418.

6. Trusolino, L., et al. 2000. HGF/scatter factor selectively promotes cell invasion by increasing integrin avidity. FASEB J. 14:1629-1640.

7. Katagiri, K., et al. 2000. Rap1 is a potent activation signal for leukocyte function-associated antigen 1 distinct from protein kinase $\mathrm{C}$ and phosphatidylinositol-3-OH kinase. Mol. Cell. Biol. 20:1956-1969.

8. Reedquist, K.A., et al. 2000. The small GTPase, Rap1, mediates CD31induced integrin adhesion. J. Cell Biol. 148:1151-1158.

9. Hadari, Y.R., et al. 2000. Galectin-8 binding to integrins inhibits cell adhesion and induces apoptosis. J. Cell Sci. 113:2385-2397.

10. Worthylake, R.A., Lemoine, S., Watson, J.M., and Burridge, K. 2001. RhoA is required for monocyte tail retraction during transendothelial migration. J. Cell Biol. 154:147-160.

11. Laukaitis, C.M., Webb, D.J., Donais, K., and Horwitz, A.F. 2001. Differential dynamics of $\alpha 5$ integrin, paxillin, and $\alpha$-actinin during formation and disassembly of adhesions in migrating cells. J. Cell Biol. 153:1427-1440.

12. Carragher, N.O., Fincham, V.J., Riley, D., and Frame, M.C. 2001. Cleavage of focal adhesion kinase by different proteases during SRC-regulated transformation and apoptosis. Distinct roles for calpain and caspases. J. Biol. Chem. 276:4270-4275.

13. O'Neill, G.M., and Golemis, E.A. 2001. Proteolysis of the docking protein HEF1 and implications for focal adhesion dynamics. Mol. Cell. Biol. 21:5094-5108.

14. Pierini, L.M., et al. 2000. Oriented endocytic recycling of $\alpha 5 \beta 1$ in motile neutrophils. Blood. 95:2471-2480. 
15. Ng, T., et al. 1999. PKC $\alpha$ regulates $\beta 1$ integrin-dependent cell motility through association and control of integrin traffic. $E M B O \mathrm{~J}$. 18:3909-3923.

16. Sieg, D.J., et al. 2000. FAK integrates growth-factor and integrin signals to promote cell migration. Nat. Cell Biol. 2:249-256.

17. Kiosses, W.B., et al. 1999. A role for p21-activated kinase in endothelial cell migration. J. Cell Biol. 147:831-844.

18. Cary, L.A., et al. 1998. Identification of p130Cas as a mediator of focal adhesion kinase-promoted cell migration. J. Cell Biol. 140:211-221.

19. Klemke, R.L., et al. 1998. CAS/Crk coupling serves as a "molecular switch" for induction of cell migration. J. Cell Biol. 140:961-972.

20. Etienne-Manneville, S., and Hall, A. 2001. Integrin-mediated activation of Cdc42 controls cell polarity in migrating astrocytes through PKC $\zeta$. Cell. 106:489-498.

21. Cox, E.A., Sastry, S.K., and Huttenlocher, A. 2001. Integrin-mediated adhesion regulates cell polarity and membrane protrusion through the Rho family of GTPases. Mol. Biol. Cell. 12:265-277.

22. Chen, R., et al. 2001. Regulation of the PH-domain-containing tyrosine kinase Etk by focal adhesion kinase through the FERM domain. Nat. Cell Biol. 3:439-444.

23. Klemke, R.L., et al. 1997. Regulation of cell motility by mitogen-activated protein kinase. J. Cell Biol. 137:481-492.

24. Fincham, V.J., James, M., Frame, M.C., and Winder, S.J. 2000. Active ERK/MAP kinase is targeted to newly forming cell-matrix adhesions by integrin engagement and v-Src. EMBO J. 19:2911-2923.

25. Wary, K.K., Mariotti, A., Zurzolo, C., and Giancotti, F.G. 1998. A requirement for caveolin-1 and associated kinase Fyn in integrin signaling and anchorage-dependent cell growth. Cell. 94:625-634.

26. Nakamoto, T., et al. 2000. CIZ, a zinc finger protein that interacts with $\mathrm{p} 130$ (cas) and activates the expression of matrix metalloproteinases. Mol. Cell. Biol. 20:1649-1658.

27. Pozzi, A., et al. 2000. Elevated matrix metalloprotease and angiostatin levels in integrin $\alpha 1$ knockout mice cause reduced tumor vascularization. Proc. Natl. Acad. Sci. USA. 97:2202-2207.

28. Dumin, J.A., et al. 2001. Pro-collagenase-1 (matrix metalloproteinase-1) binds the $\alpha 2 \beta 1$ integrin upon release from keratinocytes migrating on type I collagen. J. Biol. Chem. 276:29368-29374.

29. Silletti, S., et al. 2001. Disruption of matrix metalloproteinase 2 binding to integrin $\alpha v \beta 3$ by an organic molecule inhibits angiogenesis and tumor growth in vivo. Proc. Natl. Acad. Sci. USA. 98:119-124.

30. Roovers, K., and Assoian, R.K. 2000. Integrating the MAP kinase signal into the G1 phase cell cycle machinery. Bioessays. 22:818-826.

31. Cook, S.J., Aziz, N., and McMahon, M. 1999. The repertoire of fos and jun proteins expressed during the G1 phase of the cell cycle is determined by the duration of mitogen-activated protein kinase activation. Mol. Cell. Biol. 19:330-341.

32. Howe, A.K., and Juliano, R.L. 2000. Regulation of anchorage-dependent signal transduction by protein kinase A and p21-activated kinase. Nat. Cell Biol. 2:593-600.

33. Faraldo, M.M., Deugnier, M.A., Thiery, J.P., and Glukhova, M.A. 2001 Growth defects induced by perturbation of $\beta 1$-integrin function in the mammary gland epithelium result from a lack of MAPK activation via the Shc and Akt pathways. EMBO Rep. 2:431-437.

34. Danen, E.H., Sonneveld, P., Sonnenberg, A., and Yamada, K.M. 2000. Dual stimulation of Ras/mitogen-activated protein kinase and RhoA by cell adhesion to fibronectin supports growth factor-stimulated cell cycle progression. J. Cell Biol. 151:1413-1422.

35. Mettouchi, A., et al. 2001. Integrin-specific activation of Rac controls progression through the G(1) phase of the cell cycle. Mol. Cell. 8:115-127.

36. Feng, L.X., Ravindranath, N., and Dym, M. 2000. Stem cell factor/c-kit up-regulates cyclin D3 and promotes cell cycle progression via the phosphoinositide 3-kinase/p70 S6 kinase pathway in spermatogonia. J. Biol. Chem. 275:25572-25576.

37. Oktay, M., et al. 1998. Integrin-mediated activation of focal adhesion kinase is required for signaling to Jun $\mathrm{NH} 2$-terminal kinase and progression through the G1 phase of the cell cycle. J. Cell Biol. 145:1461-1469.

38. Matter, M.L., and Ruoslahti, E. 2001. A signaling pathway from the alpha5beta 1 and $\alpha v \beta 3$ integrins that elevates bcl- 2 transcription. J. Biol. Chem. 276:27757-27763.

39. Persad, S., et al. 2001. Regulation of protein kinase B/Akt-serine 473 phosphorylation by integrin-linked kinase: critical roles for kinase activity and amino acids arginine 211 and serine 343. J. Biol. Chem. 276:27462-27469.

40. Cho, S.Y., and Klemke, R.L. 2000. Extracellular-regulated kinase activation and CAS/Crk coupling regulate cell migration and suppress apoptosis during invasion of the extracellular matrix. J. Cell Biol. 149:223-236.

41. Lee, J.W., and Juliano, R.L. 2000. $\alpha 5 \beta 1$ integrin protects intestinal epithelial cells from apoptosis through a phosphatidylinositol 3-kinase and protein kinase B-dependent pathway. Mol. Biol. Cell. 11:1973-1987.

42. Almeida, E.A., et al. 2000. Matrix survival signaling: from fibronectin via focal adhesion kinase to c-Jun $\mathrm{NH}(2)$-terminal kinase. J. Cell Biol. 149:741-754.

43. Puthalakath, H., et al. 2001. Bmf: a proapoptotic BH3-only protein regulated by interaction with the myosin $\mathrm{V}$ actin motor complex, activated by anoikis. Science. 293:1829-1832.

44. Baeckstrom, D., Lu, P.J., and Taylor-Papadimitriou, J. 2000. Activation of the $\alpha 2 \beta 1$ integrin prevents c-erbB2-induced scattering and apoptosis of human mammary epithelial cells in collagen. Oncogene. 19:4592-4603.

45. Bachelder, R.E., et al. 1999. p53 inhibits $\alpha 6 \beta 4$ integrin survival signaling by promoting the caspase 3 -dependent cleavage of AKT/PKB. J. Cell Biol. 147:1063-1072

46. Boube, M., Martin-Bermudo, M.D., Brown, N.H., and Casanova, J. 2001. Specific tracheal migration is mediated by complementary expression of cell surface proteins. Genes Dev. 15:1554-1562.

47. Kassis, J., Lauffenburger, D.A., Turner, T., and Wells, A. 2001. Tumor invasion as dysregulated cell motility. Semin. Cancer Biol. 11:105-117.

48. Sethi, T., et al. 1999. Extracellular matrix proteins protect small cell lung cancer cells against apoptosis: a mechanism for small cell lung cancer growth and drug resistance in vivo. Nat. Med. 5:662-668.

49. Aoudjit, F., and Vuori, K. 2001. Integrin signaling inhibits paclitaxelinduced apoptosis in breast cancer cells. Oncogene. 20:4995-5004. 